\title{
Gel spacer to protect carotid artery and reconstructed jejunum in image-guided interstitial brachytherapy for recurrent hypopharyngeal cancer: a technical report
}

\author{
Naoya Murakami, MD, PhD', Yoshitaka Honma, MD², Seiichi Yoshimoto, MD, PhD³, Satoshi Shima, MD', \\ Tairo Kashihara, MD, PhD', Kana Takahashi, MD, PhD', Tomoya Kaneda, MD, PhD', Koji Inaba, MD, PhD!, \\ Kae Okuma, MD, PhD', Koji Masui, MD, PhD, Ken Yoshida, MD, PhD ${ }^{5}$. Hiroshi lgaki, MD, PhD', Jun Itami, MD, PhD' \\ 'Department of Radiation Oncology, National Cancer Center Hospital, Tokyo, Japan, ${ }^{2}$ Head and Neck Medical Oncology Division, \\ National Cancer Center Hospital, Japan, ${ }^{3}$ Department of Head and Neck Surgery, National Cancer Center Hospital, Japan, ${ }^{4}$ Department \\ of Radiology, Graduate School of Medical Science, Kyoto Prefectural University of Medicine, Japan, ${ }^{5}$ Department of Radiology, Kansai \\ Medical University, Hirakata, Japan
}

\begin{abstract}
In this technical report, patient with total pharyngolaryngectomy and jejunum interposition experienced lymph node metastasis in the reconstructed mesenteric lymph nodes. Because this patient received 45 Gy of whole neck radiation therapy 24 years ago and small bowel's tolerance dose of the reconstructed jejunum was considered to be low, spacer gel injection was applied during image-guided interstitial brachytherapy to decrease doses to the reconstructed jejunum and previously irradiated carotid artery.

J Contemp Brachytherapy 2021; 13, 5: 583-587 DOI: https://doi.org/10.5114/jcb.2021.109806
\end{abstract}

Key words: hypopharyngeal cancer, interstitial brachytherapy, image-guided brachytherapy, gel spacer.

\section{Purpose}

Gel spacer has been used to create a space between clinical target volume (CTV) and organs at risk (OARs) to protect them from toxic high-dose radiation exposure in radiation therapy of prostate cancer or gynecologic malignancies. In a phase III clinical trial, its protective property for rectal toxicities was demonstrated in external beam radiation therapy for prostate cancer. Likewise, our previous reports have shown that the gel spacer could reduce rectal and bladder dose and reduce frequency of late rectal bleeding [1-4]. However, to the best of our knowledge, it has not been used for head and neck radiation therapy. In this case report, we presented a case with previous whole neck irradiation and developed lymph node metastasis in the reconstructed jejunum mesenteric lymph nodes, who received palliative image-guided interstitial brachytherapy (IG-ISBT) to obtain local control. Because this patient already had received 45 Gy of whole neck radiation therapy 24 years ago, and the reconstructed jejunum should be protected due to small bowel's tolerance dose being relatively low, spacer gel injection was utilized during IG-ISBT to protect these OARs from highdose radiation exposure (complication probability of $5 \%$ after 5 years (TD 5/5) for late toxicity at a dose of $50 \mathrm{~Gy}$ for irradiating $1 / 3$ of the small bowel was reported [5]).

Written informed consent was obtained from the patients for spacer gel injection. This case report was approved by the Institutional Review Board of National Cancer Center Hospital, with Number 2017-091, according to ethical standards of the Declaration of Helsinki.

\section{Case description}

A 72-year-old male, with a previous history of $45 \mathrm{~Gy}$ in 18 fractions of bilateral whole neck radiation therapy (level I-V) performed using a single antero-posterior-oriented cobalt-60 beam irradiation 24 years ago for malignant lymphoma, and suffered from T3N0M0 hypopharyngeal squamous cell carcinoma in 2004. He underwent total pharyngolaryngectomy and bilateral neck dissection with jejunum interposition. Ten years later, the patient experienced bilateral regional lymph node metastasis, including mesenteric lymph node in the re- 
constructed jejunum, and received total removal of the reconstructed jejunum and metastatic neck lymph nodes with secondary jejunum reconstruction. Three years later, the patient experienced regional lymph node metastasis, including mesenteric lymph node in the secondary reconstructed jejunum, and received total removal of the reconstructed jejunum and metastatic neck lymph nodes with tertiary jejunum reconstruction. Two years later, the patient experienced third-time regional lymph node metastasis, which was confirmed as squamous cell carcinoma by fine-needle aspiration cytology. The patient received six cycles of a combination of 5-fluorouracil/ carboplatin/cetuximab (5 FU/CBDCA/C-mab), which resulted in a partial response. However, eight months after systemic chemotherapy initiation, progressive dis- ease was noted, and nivolumab was started. Six months after the start of nivolumab, positron emission tomography-computed tomography (PET-CT) showed recurrent disease in the head and neck region, including bilateral level $\mathrm{V}$ and multiple mesenteric lymph nodes involvement (Figure 1). At this point, further surgical treatment was not planned, and systemic chemotherapy composed of a combination of $5 \mathrm{FU} / \mathrm{CBDCA} / \mathrm{C}-\mathrm{mab}$ was started. Six cycles of $5 \mathrm{FU} / \mathrm{CBDCA} / \mathrm{C}$-mab resulted in a partial response; however, seven months after the initiation of $5 \mathrm{FU} / \mathrm{CBDCA} / \mathrm{C}-\mathrm{mab}$, progressive disease was noted and subsequently, nivolumab was started. Five months after the initiation of nivolumab, during its $9^{\text {th }}$ cycle, the patient felt discomfort with persistent mesenteric lymph nodes (Figure 1), and wanted to receive palliative radi-

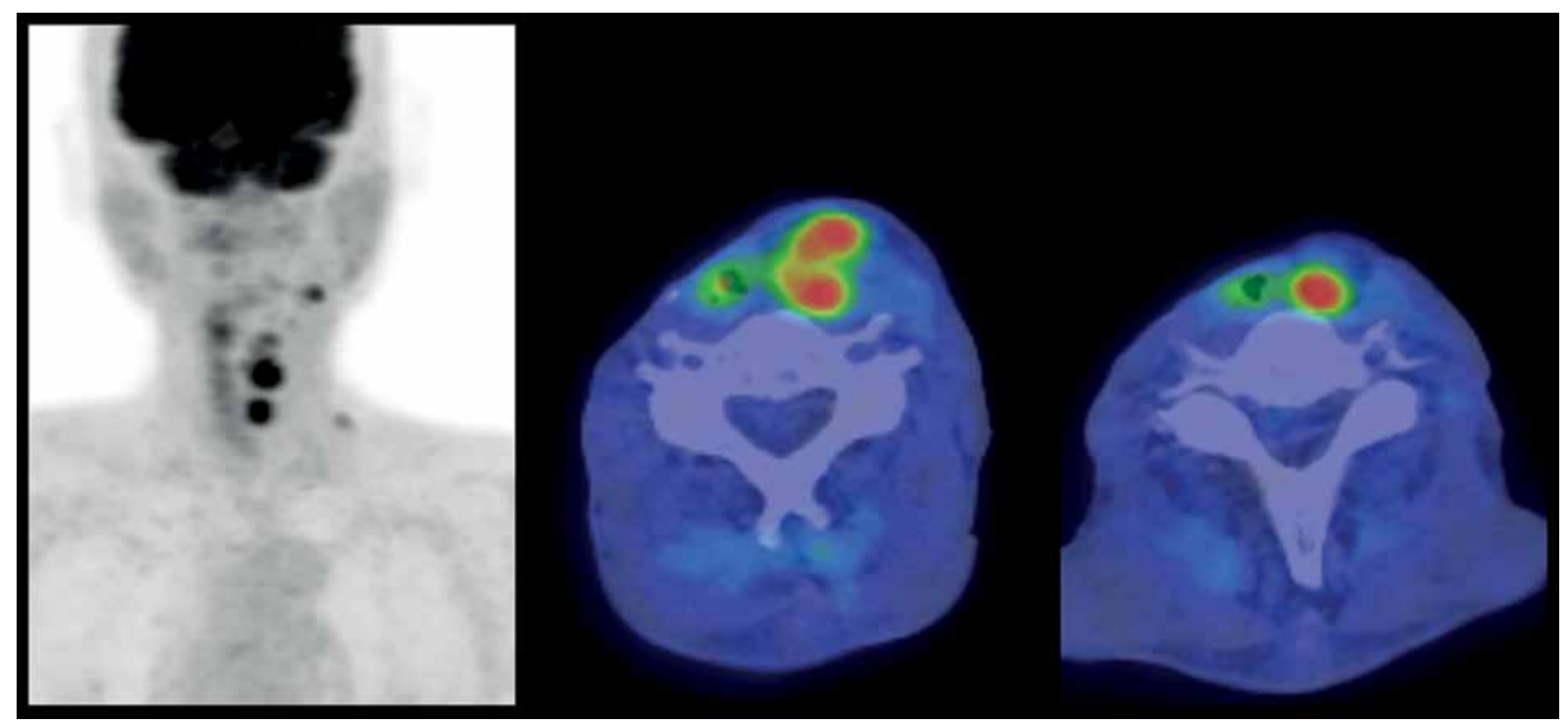

Fig. 1. Fluorodeoxyglucose (FDG)-PET-CT images before salvage high-dose-rate interstitial brachytherapy for mesenteric lymph nodes of reconstructed jejunum. Red color represents high, and blue color represents low uptake on the color scale

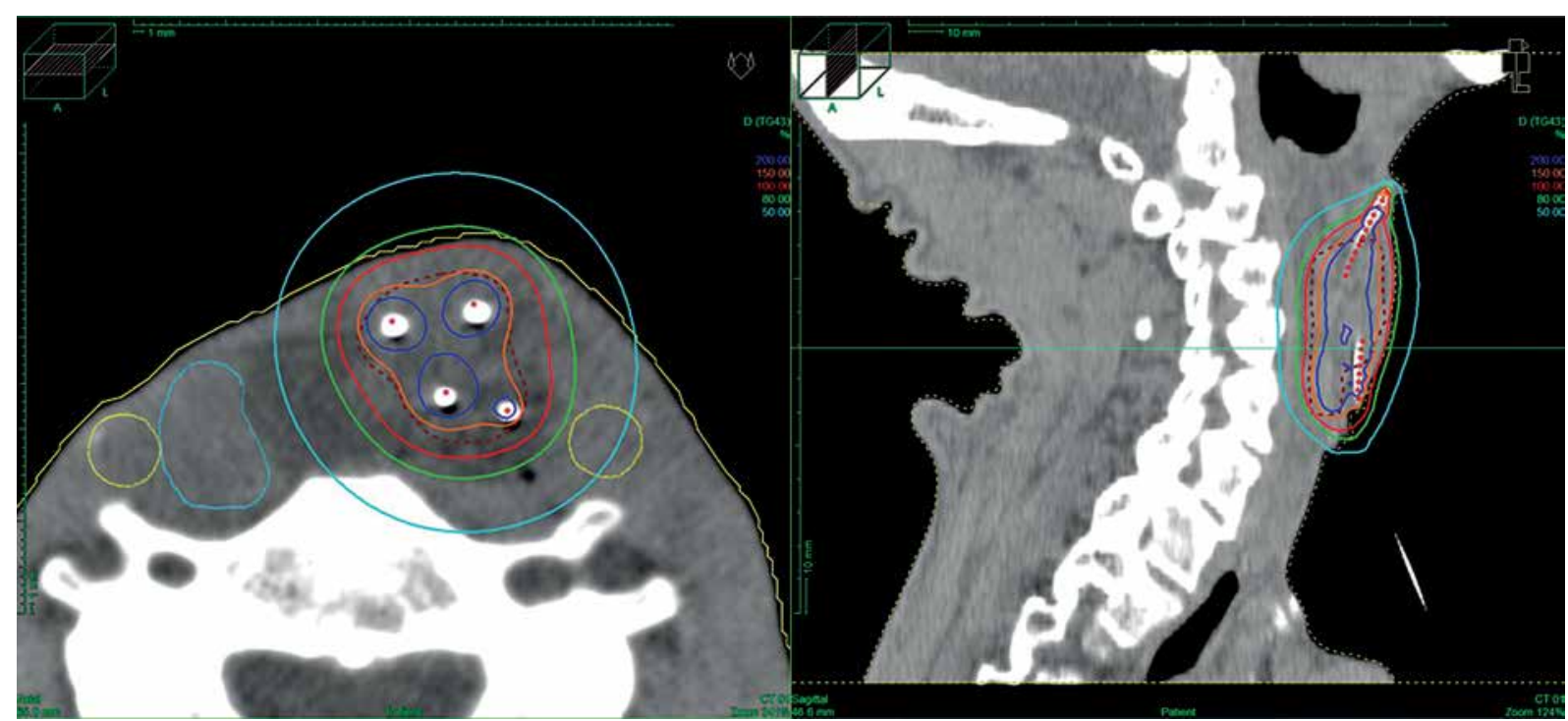

Fig. 2. Dose distribution of high-dose-rate interstitial brachytherapy. Blue, red, green, and sky-blue lines represent $200 \%, 100 \%$, $80 \%$, and $50 \%$ isodose lines, respectively. The light blue object on the right neck represents reconstructed jejunum, yellow circles show carotid arteries, and red dotted line indicate clinical target volume 
ation therapy to control metastatic lymph nodes. Although the larynx was previously totally removed because he already received $45 \mathrm{~Gy}$ of whole neck irradiation 24 years ago, high-dose-rate interstitial brachytherapy (HDR-ISBT) was selected, trying to confine radiation exposure to OARs surrounding the target volume as little as possible. The prescription dose per fraction was $7 \mathrm{~Gy}$ $\left(D_{90}>7\right.$ Gy), in which $100 \%$ isodose line covered the CTV, and irradiation was performed twice daily at 6-hour intervals (Figure 2). A total of $42 \mathrm{~Gy}$ in 6 fractions were delivered in 3 days with needle inserted in place for 3 days. Needle insertion was performed under ultrasonography guidance, so that the needles pierced all enlarged nodes, and 5-French ProGuide ${ }^{\circledR}$ sharp plastic needles (Nucletron, an Elekta Company, Elekta AB, Stockholm, Swe- den) were used. Because the enlarged mesenteric lymph nodes were located between the reconstructed jejunum in the right side and left carotid artery, a hyaluronic acid gel spacer (Suvenyl ${ }^{\circledR}$, Chugai Pharmaceutical Co., Tokyo, Japan) was applied to create a distance between high-dose area and OARs, including the reconstructed jejunum and the left carotid artery (Figure 3). CTV $D_{90}$ was $9.0 \mathrm{~Gy}$ and CTV $\mathrm{V}_{100}$ was $99.3 \%$, while the reconstructed jejunum $\mathrm{D}_{1 \mathrm{cc}}$ and left carotid artery $\mathrm{D}_{1 \mathrm{cc}}$ were 2.4 and 3.8 Gy, respectively. The gel spacer injection was performed once per day in the morning fraction under ultrasound guidance. No acute radiation-induced side effect was noted, apart from grade 1 dermatitis. PET-CT performed three months after the completion of HDR-ISBT resulted in a complete metabolic response (Figure 4).

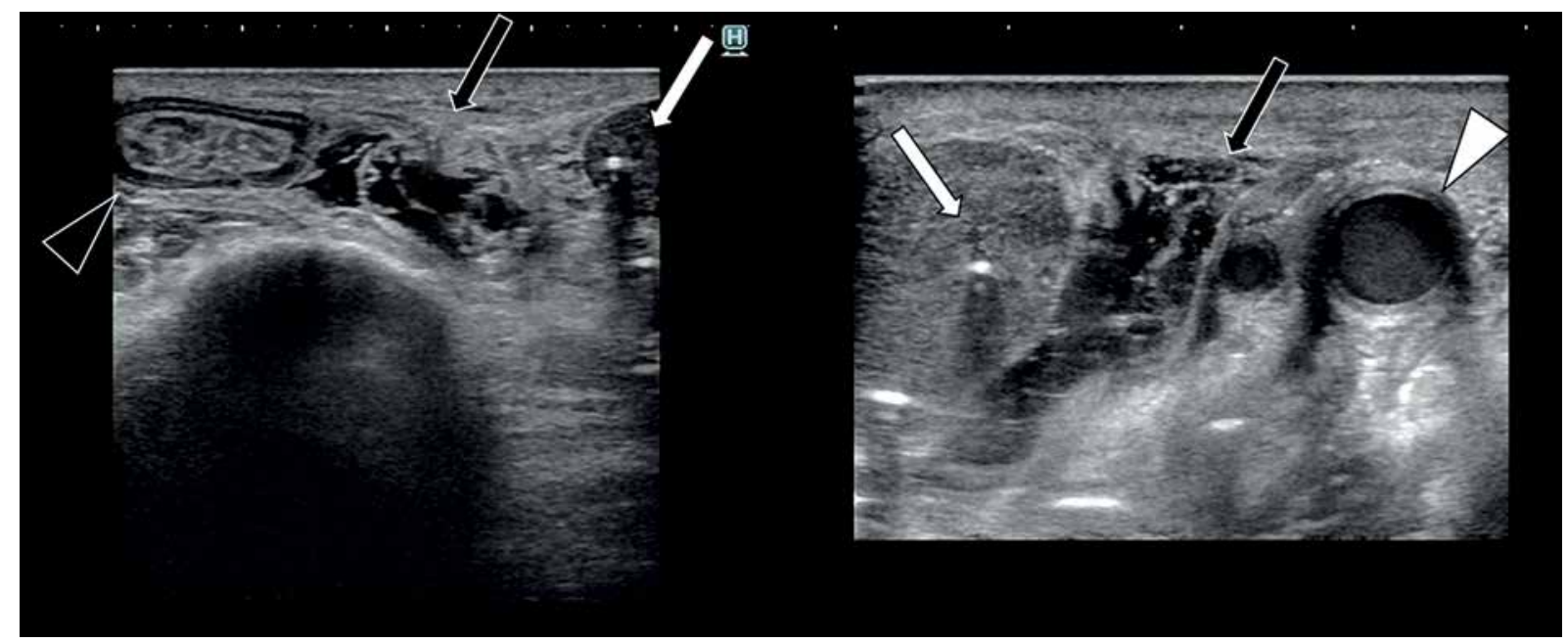

Fig. 3. Axial ultrasonography images. Needle insertion was performed under ultrasonography guidance. Four needles (ProGuide $^{\circledR}$ sharp plastic needle, Nucletron, an Elekta Company, Elekta AB, Stockholm, Sweden) were inserted to irradiate metastatic mesenteric lymph nodes. White arrow represents metastatic mesenteric lymph node, black arrow indicates gel spacer, white arrowhead shows left carotid artery, and black arrowhead represents reconstructed jejunum

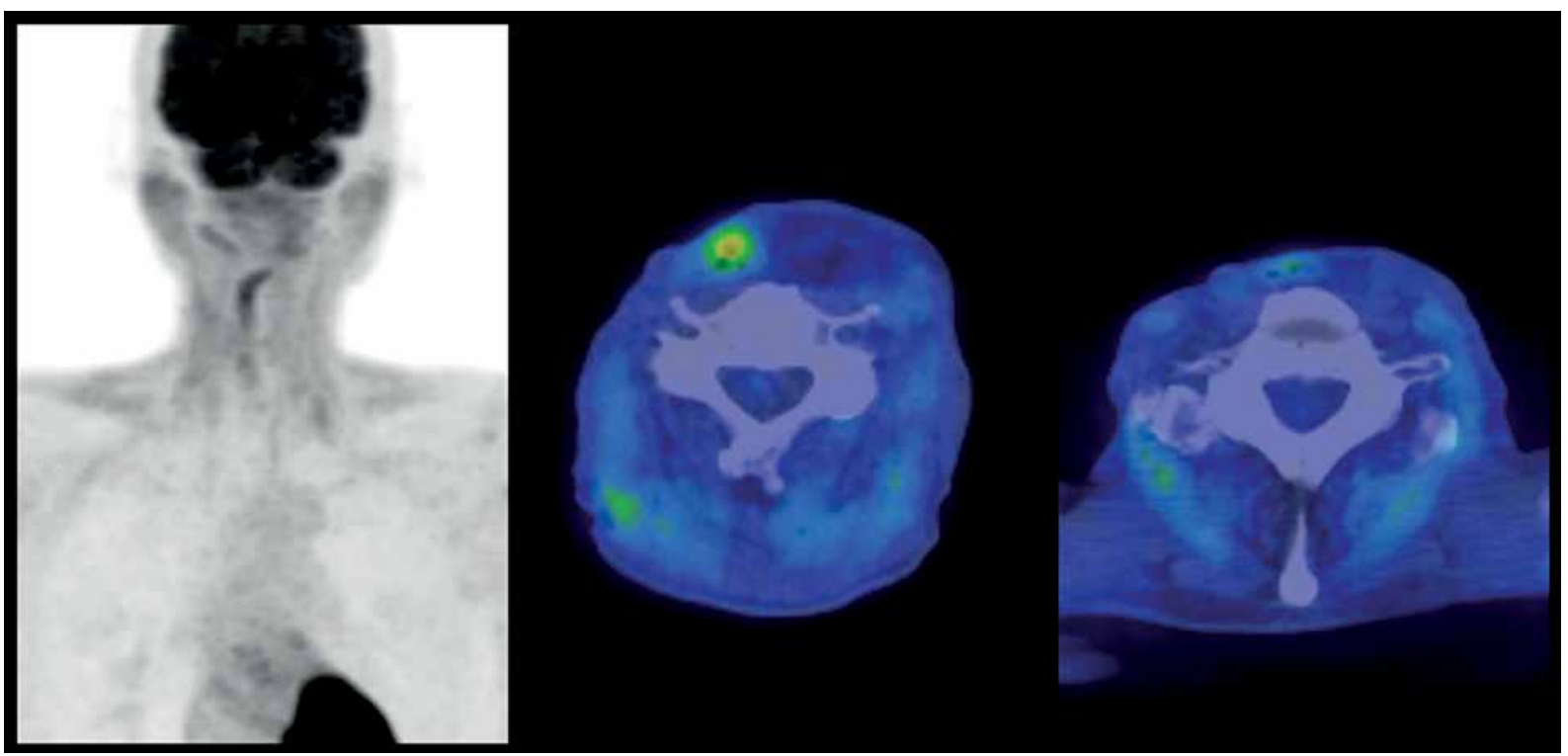

Fig. 4. Fluorodeoxyglucose (FDG)-PET-CT images 3 months after salvage high-dose-rate interstitial brachytherapy for mesenteric lymph nodes of reconstructed jejunum 


\section{Discussion}

Gel spacer has been utilized to create a space between CTV and OARs in both external beam radiation therapy (EBRT) and brachytherapy (BRT) [1-4, 6-9]. In a phase III clinical trial, it was demonstrated that SpaceOAR ${ }^{\mathrm{TM}}$ (Augmenix, Inc., Waltham, MA, USA), polyethylene-glycol hydrogel spacer could reduce rectal dose and rectal toxicity [8]. Our group has been using hyaluronate spacer gel Suvenyl ${ }^{\mathrm{TM}}$ (Chugai/Roche, Tokyo, Japan) for gynecologic BRT, and reported its usefulness in reducing rectal dose [1], bladder dose [2], and frequency of rectal bleeding [4]. By physically creating a space between CTV and OARs, it is possible to deliver adequate tumoricidal dose to CTV, while reducing doses to OARs. Moreover, it is possible to raise the likelihood of cure by widening a therapeutic window of tumor control.

The head and neck region is also the appropriate anatomical area for applying BRT, either definitive or in combination with external beam radiation therapy [10-21] However, unlike pelvic organs, only a small amount of soft tissue is available in the head and neck region. Therefore, it is less likely that the gel spacer is utilized for organ protection in head and neck BT. Kishi et al. previously reported that gel spacer could be used to shift critical OARs from surrounding target volume in re-irradiation settings for 30 patients after full-dose radiation therapy, including 13 head and neck cancer patients [6]. In line with this report, gel spacer effectively shifted critical OARs in the current case. The prescription dose of 7 Gy per fraction was selected because it was anticipated that gel spacer could push the reconstructed jejunum and carotid artery away from high-dose area. Dose per fraction lower than 4 Gy could be considered, but since there were so many patients who needed BRT, there was no room for multiple treatment sessions for this patient. Therefore, a short treatment schedule was selected due to logistic reasons.

The limitations of this report is that there was no CT without gel spacer; accordingly, there was no direct comparison of OARs' doses between those with and without gel spacer. However, due to BRT's nature of steep dose fall-off close to the sources, it was evident that by simply creating a small gap between a radioactive source and OARs, it will reduce OARs' radiation exposure considerably. Additionally, only dosimetric results were shown, and no long-term clinical consequence was provided in this report. Therefore, further follow-up and more cases with similar clinical scenarios are mandatory to obtain long-term clinical advantages of this technique. To the best of our knowledge, this was the first report on applying gel spacer to protect the reconstructed jejunum after total laryngectomy. Due to the complex anatomy of the head and neck region, with only a small amount of fat and soft tissues surrounding OARs, it was generally difficult to apply gel spacer to protect OARs. However, when applicable, gel spacer plays a vital role in delivering tumoricidal dose, while decreasing doses to OARs' to prevent late radiation-related toxicities. Further studies are needed to establish the role of gel spacer in managing head and neck malignancies.

\section{Funding}

This work was in part financially supported by the Japan Agency for Medical Research and Development (AMED) and the National Cancer Center Research and Development Fund (26-A-18 and 26-A-28).

\section{Consent for publication and ethics approval and consent to participate}

We obtained a written informed consent from the patient for publication including each clinical datum as images, case history, and other clinical data. This case report was approved by the Institutional Review Board of the National Cancer Center Hospital (approval Number, 2017-091), according to the ethical standards laid down in the Declaration of Helsinki.

\section{Disclosure}

Dr. Inaba obtains grants from Elekta outside the submitted work. Dr. Igaki acquires grants from HekaBio and receives personal fee from Itochu outside the submitted work. Dr. Nakayama receives personal fees from Astra Zeneca outside the submitted work. Dr. Itami receives personal fees from HekaBio and from kay J outside the submitted work. The rest of the authors have no conflicts of interests to declare.

\section{References}

1. Kashihara T, Murakami N, Tselis N et al. Hyaluronate gel injection for rectum dose reduction in gynecologic high-doserate brachytherapy: initial Japanese experience. J Radiat Res 2019; 60: 501-508.

2. Murakami N, Shima S, Kashihara T et al. Hyaluronic gel injection into the vesicovaginal septum for high-dose-rate brachytherapy of uterine cervical cancer: an effective approach for bladder dose reduction. J Contemp Brachytherapy 2019; 11: 1-7.

3. Iijima K, Murakami N, Nakamura S et al. Configuration analysis of the injection position and shape of the gel spacer in gynecologic brachytherapy. Brachytherapy 2021; 20: 95-103.

4. Murakami N, Nakamura S, Kashihara T et al. Hyaluronic acid gel injection in rectovaginal septum reduced incidence of rectal bleeding in brachytherapy for gynecological malignancies. Brachytherapy 2020; 19: 154-161.

5. Emami B, Lyman J, Brown A et al. Tolerance of normal tissue to therapeutic irradiation. Int J Radiat Oncol Biol Phys 1991; 21: 109-122.

6. Kishi K, Sonomura T, Shirai S et al. Critical organ preservation in reirradiation brachytherapy by injectable spacer. Int J Radiat Oncol Biol Phys 2009; 75: 587-594.

7. Pinkawa M, Corral NE, Caffaro $M$ et al. Application of a spacer gel to optimize three-dimensional conformal and intensity modulated radiotherapy for prostate cancer. Radiother Oncol 2011; 100: 436-441.

8. Mariados N, Sylvester J, Shah D et al. Hydrogel spacer prospective multicenter randomized controlled pivotal trial: dosimetric and clinical effects of perirectal spacer application in men undergoing prostate image guided intensity modulated radiation therapy. Int J Radiat Oncol Biol Phys 2015; 92: 971-977.

9. Tang Q, Zhao F, Yu X et al. The role of radioprotective spacers in clinical practice: a review. Quant Imaging Med Surg 2018; 8: 514-524. 
10. Masui K, Yamazaki H, Suzuki G et al. High dose rate interstitial brachytherapy for early stage lip cancer using customized dental spacer. J Radiat Res 2020; 61: 506-510.

11. Murakami N, Cheng G, Yoshimoto S, Itami J. Image-guided interstitial brachytherapy boost for nasopharyngeal carcinoma: technical aspects. J Contemp Brachytherapy 2020; 12: 294-302.

12. Murakami N, Ueno T, Yatsuoka $W$ et al. Dose coverage comparison between "interstitial catheter-only" and "hybrid intracavitary-interstitial brachytherapy" for early stage squamous cell carcinoma of the buccal mucosa. J Contemp Brachytherapy 2018; 10: 486-491.

13. Murakami N, Yoshimoto S, Nakamura S et al. Per-oral interstitial brachytherapy catheter insertion for boost in case of recurrent tonsillar carcinoma: dosimetry and clinical outcome. BJR Case Rep 2020; 6: 20190059.

14. Murakami N, Yoshimoto S, Uematsu M et al. Image-guided interstitial brachytherapy boost for keratinizing squamous cell carcinoma of inferior wall of the nasopharynx. BJR Case Rep 2020; 6: 20200005.

15. Takagawa Y, Murakami N, Suzuki S et al. High-dose-rate interstitial brachytherapy for a bulky sebaceous carcinoma of the eyelid: A case report. Clin Case Rep 2019; 7: 1844-1848.

16. Yoshida K, Kotsuma T, Akiyama $\mathrm{H}$ et al. A new implant device to prevent edema-associated underdosage in high-doserate interstitial brachytherapy of mobile tongue cancer. J Contemp Brachytherapy 2019; 11: 573-578.

17. Takacsi-Nagy Z, Oberna F, Koltai P et al. Long-term outcomes with high-dose-rate brachytherapy for the management of base of tongue cancer. Brachytherapy 2013; 12: 535-541.

18. Takacsi-Nagy Z, Martinez-Mongue R, Mazeron JJ et al. American Brachytherapy Society Task Group Report: Combined external beam irradiation and interstitial brachytherapy for base of tongue tumors and other head and neck sites in the era of new technologies. Brachytherapy 2017; 16: 44-58.

19. Tagliaferri L, Bussu F, Rigante M et al. Endoscopy-guided brachytherapy for sinonasal and nasopharyngeal recurrences. Brachytherapy 2015; 14: 419-425.

20. Pernot M, Hoffstetter S, Peiffert D et al. Role of interstitial brachytherapy in oral and oropharyngeal carcinoma: reflection of a series of 1344 patients treated at the time of initial presentation. Otolaryngol Head Neck Surg 1996; 115: 519-526.

21. Kovacs G, Martinez-Monge R, Budrukkar A et al. GEC-ESTRO ACROP recommendations for head \& neck brachytherapy in squamous cell carcinomas: 1st update - Improvement by cross sectional imaging based treatment planning and stepping source technology. Radiother Oncol 2017; 122: 248-254. 